\title{
Segmentation and 3D Visualization of Medical Image : An Overview
}

\author{
Jiwoo Kang, Doyoung Kim, Sanghoon Lee \\ Department of Electrical and Electronic Engineering, College of Engineering, Yonsei University, Seoul, Korea
}

In this paper, an overview of segmentation and $3 \mathrm{D}$ visualization methods are presented. Commonly, the two kinds of methods are used to visualize organs and vessels into 3D from medical images such as CT(A) and MRI - Direct Volume Rendering (DVR) and Iso-surface Rendering (IR). DVR can be applied directly to a volume. It directly penetrates through the volume while it determines which voxels are visualizedbased on a transfer function. On the other hand, IR requires a series of processes such as segmentation, polygonization and visualization. To extract a region of interest (ROI) from the medical volume image via the segmentation, some regions of an object and a background are required, which are typically obtained from the user. To visualize the extracted regions, the boundary points of the regions should be polygonized. In other words, the boundary surface composed of polygons such as a triangle and a rectangle should be required to visualize the regions into $3 \mathrm{D}$ because illumination effects, which makes the object shaded and seen in 3D, cannot be applied directly to the points.

Key Words Medical Image Processing · Segmentation · Polygonization · 3D Visualization · Direct Volume Rendering • Iso-surface Rendering.

Received: July 7, 2014 / Revised: July 11, 2014 / Accepted: July 15, 2014

Address for correspondence: Sang Hoon Lee

Department of Electrical and Electronic Engineering, Yonsei University College of Medicine, 50 Yonsei-ro, Seodaemun-gu, Seoul, Korea

Tel: 02-2123-2767, Fax: 02-313-2879, E-mail: slee@yonsei.ac.kr

\section{서 론}

$\mathrm{CT}(\mathrm{A}), \mathrm{MRI}$ 와 같은 3차원 의료영상으로부터 혈관, 장기와 같은 의미 있는 영역을 추출하고 가시화하기 위한 많은 연구들 이 진행되어왔다. ${ }^{1-4}$ 이러한 3 차원 가시화 기술들은 의학에서 교 육 용도로 널리 이용되어 왔으며, 더 나아가 전문의가 장기 및 혈관의 배치 및 구조를 3 차원 상에서 쉽게 확인 가능하게 하여, 수술 전 수술 대상의 해부학적 특징을 미리 확인함으로써 수술 시간과 출혈량을 줄여 수술의 질적 향상을 이루어왔다. ${ }^{5-10}$

관심 영역을 가시화 하는 방법으로는 크게 직접 볼륨 렌더링 (Direct Volume Rendering)과 등위면 렌더링 (Iso-surface Rendering) 2가지로 분류할 수 있다. ${ }^{11,12}$ 직접 볼륨 렌더링은 볼륨을 직접 투과하면서 미리 정의된 전달 함수 (Transfer Function)에 따라서 어떤 복셀 (Voxel)을 가시화할 지를 결정 하여 3 차원으로 가시화한다. ${ }^{13}$ 전달 함수에는 복셀의 값 (Intensity) 또는 미분 값 (Gradient) 등과 같은 특징에 따라 해당
복셀이 가시화 될 색상과 투명도를 결정한다. 따라서 직접 볼륨 렌더링은 전달 함수를 이용하여 특정 장기나 혈관만을 3 차원으 로 가시화하는 것이 가능하므로, 가시화하길 원하는 개체의 특 징을 잘 반영할 수 있는 전달 함수를 설계하는 것이 중요하다. 등위면 렌더링을 사용 할 경우 반드시 가시화하기 전 미리 대상 개체를 의료 볼륨으로부터 추출해야 하며, 추출된 영역의 경계 면을 폴리곤 (Polygon)으로 변환해야 하는 과정이 필수적이다. 14 이는 분할 기술을 사용하여 추출된 개체의 표면은 점으로 이 루어졌는데, 점에는 3 차원 입체감을 형성해주는 조명효과 (Illumination)을 적용할 수 없기 때문에, 그래픽 하드웨어가 표현 하는 개체 표면 점들을 기본 폴리곤인 삼각형이나 사각형으로 재구성해줘야 한다. ${ }^{15,16}$ 이후 경우에 따라 개체의 표면을 구성하 는 폴리곤 집단을 개체의 곡률 등을 반영해 폴리곤의 개수와 밀 집도를 조절해주는 재구성 (Meshing) 작업을 수행한다. ${ }^{17,18}$ 이 후 원하는 위치에 조명을 만들고 그래픽 하드웨어를 사용하여 가시화할 경우 관심 개체가 3 차원으로 가시화된다. 
본 논문에서는 이러한 볼륨으로부터 관심 개체를 3차원으로 가시화하는 일련의 방법에 대해서 소개하는 바이다.

\section{Direct Volume Rendering}

직접 볼륨 렌더링은 볼륨으로부터 원하는 개체를 3차원으로 가시화하는 대표적인 방법이다 (Fig. 1). 복셀들로 이루어진 3차 원 이미지 볼륨에 광선을 쏘아, 광선이 지나는 각 복셀에서 나 온 색상과 투명도를 누적하고 계산하여 최종 색상을 결정한다 (Fig. 2). 직접 볼륨 렌더링에서는 3차원 이미지로부터 재구성된 2 차언 이미지 픽셀의 색깔을 결정하는데 볼륨을 지나는 광선 상의 모든 복셀들이 관여하기 때문에, 의료 영상 촬영 시 발생 한 노이즈에 의한 소수 복셀들의 오차는 결과에 큰 영향을 미치 지 않는다. 광선이 지나는 각 복셀의 색상을 결정하는 것은 전 달 함수로, 전달 함수는 해당 개체의 특징에 따라 미리 정의된 다. 가장 널리 사용되는 전달함수로는 1 차원 전달함수와 2 차원 전달함수가 있으며, 1 차원 전달 함수는 해당 위치의 복셀 값에 따라 색상과 투명도를 결정하며, 2차원 전달함수는 복셀 값과 그 미분 값 2 가지에 따라 결정한다. 이러한 전달함수는 혈관, 장 기 등 의료 영상 내에서는 대체로 개체간 값의 차이가 분명하기
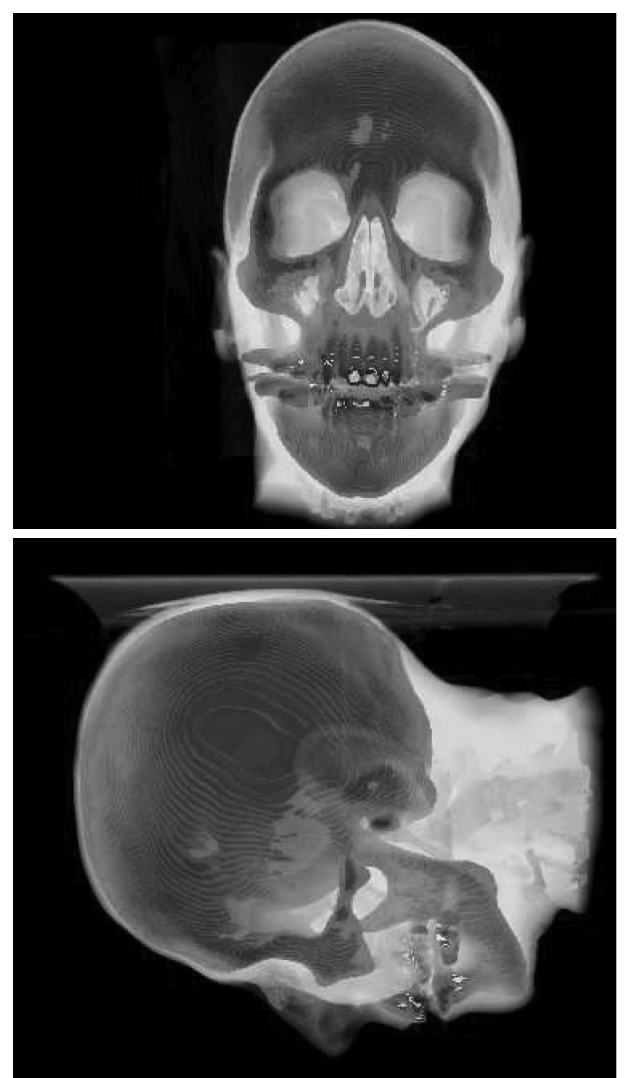

Fig. 1. 직접 볼륨 렌더링을 두개골 CT 영상에 적용해본 결과.
때문에 의료 이미지 볼륨을 효과적으로 가시화할 수 있다. 투명 도는 해당 색상의 반영 비율을 결정한다. 예를 들어 피부 부분 의 투명도가 높고, 뼈 부분의 투명도가 낮을 경우에는 피부는 반투명하게 보이며 뼈는 불투명하게 보이게 된다 (Fig. 3). 이러 한 투명도를 사용함으로써 인체 내부의 여러 개체를 계층적으 로 확인 가능하며, 이러한 특징 때문에 의학 분야에서는 진단용 으로 직접 볼륨 렌더링을 널리 이용하고 있다.

\section{Iso-surface Rendering}

등위면 렌더링은 직접 볼륨 렌더링과는 달리 3 차원 볼륨으로 부터 원하는 개체의 영역을 추출하는 분할 과정, 그리고 추출된 영역의 경계점들을 면으로 바꿔주는 폴리건화 (Polygonization) 과정 후 가시화가 수행된다.

3 차원 볼륨으로부터 원하는 개체의 영역을 추출하기 위해서 는 분할 (Segmentation) 기술이 사용되는데, 의료 영상에서 가 장 많이 사용되는 분할 기술로는 Region Growing, Active Contour, Level Set 그리고 Graph-cut 기법이 있다. ${ }^{20} \mathrm{Re}-$ gion Growing은 사용자로부터 하나의 시작점 (Seed)을 입력

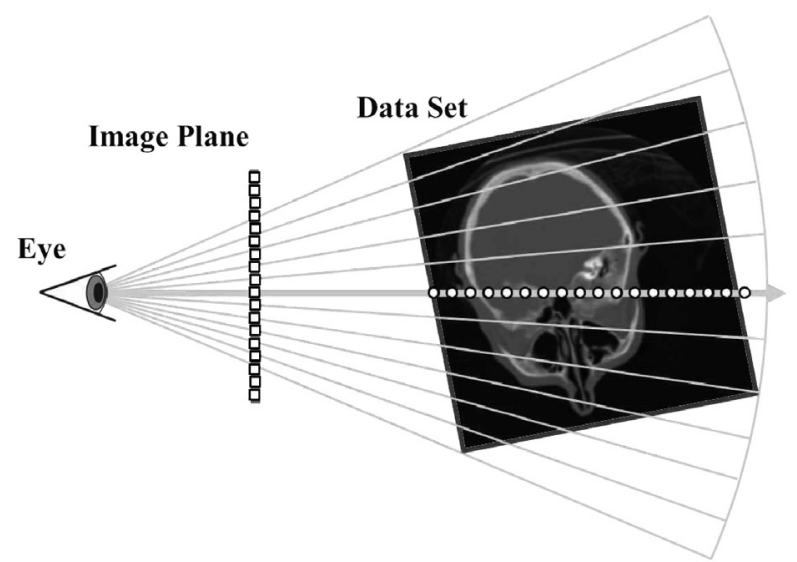

Fig. 2. 직접 볼륨 렌더링에서는 이미지 각 필셀에서 볼륨으로부터 광 선을 쏘아 광선이 지나는 각 점의 특징에 따라 색상을 결정한다.
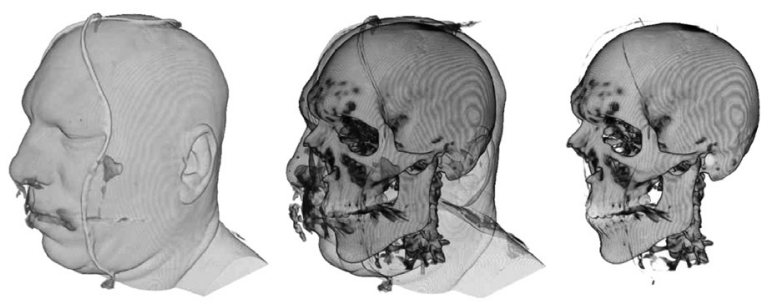

Fig. 3. 직접 볼륨 렌더링은 전달 함수를 어떻게 정의하느냐에 따라 가시화하는 대상이 달라지며, 투명도를 이용할 경우 피부-뼈 와 같이 계층 구조에 있는 개체들도 동시에 표현 가능하다. ${ }^{19}$ 
받아, 이웃 픽셀들로 확장해나간다 (Fig. 4). 지금까지 확장된 영역과 이웃된 픽셀이 서로 유사할 경우 해당 픽셀로 확장해나 가고, 유사하지 않을 경우 해당 픽셀로는 더 이상 확장해나가지 않는다. 따라서 Region Growing은 유사도를 결정하는 함수의 정의가 가장 성능에 큰 영향을 미치며 보통 확장된 영역의 평균 값 (Average Intensity)이나 분산 등을 이용해서 정의된다. Region Growing은 의료 분야에서 가장 많이 사용되는 분할 기법이긴 하나, 실제로 같은 개체가 아님에도 노이즈에 의해서 발생한 픽셀을 통해 확장되어 과분할 (Over-segmentation) 현상이 발생하기 쉬어, 영상전문의에 의해 후처리가 반드시 필요 하다. Active Contour는 Snake라고도 불리며 사용자로부터 개체 주위의 점들을 입력 받아 해당 점들이 에너지가 최소화 되 는 방향으로 움직여서 개체를 분할하는 방식이다. 입력 받은 점 들은 Spline과 같은 보간 함수에 의해 곡선으로 근사하게 된다. 에너지 함수는 보통 보간 된 곡선의 곡률, 길이와 같이 곡선 내 부적인 요소와 해당 점이 있는 위치의 픽셀 값, 차분 값 (Gradient)와 같은 곡선 외부적인 요소로 구성된다. 예를 들어 혈관 을 분할한다고 가정해보자. 곡률이 완만할 수록, 곡선의 길이가 짧을수록. 픽셀 값이 밝을수록, 차분 값이 클수록 에너지 함수 가 작아진다고 하면 혈관 경계면에서 점들의 에너지가 최소화 되게 된다 (Fig. 5). Level-set은 Active Contour와 유사하지 만, 점들이 에너지가 최소가 되는 방향으로 움직인다면 Levelset은 Implicit Level Set 함수가 반복할 때마다 에너지가 최소 화 하는 방향으로 움직인다. Implicit Level Set 함수가 3차원 함수 라고 하면, $\mathrm{z}$ 가 상수로 고정되면 $\mathrm{x}, \mathrm{y}$ 는 2 차원 경계면을 그리게 된다. Level Set 분할 방법에서는 z가 0일 때 Implicit
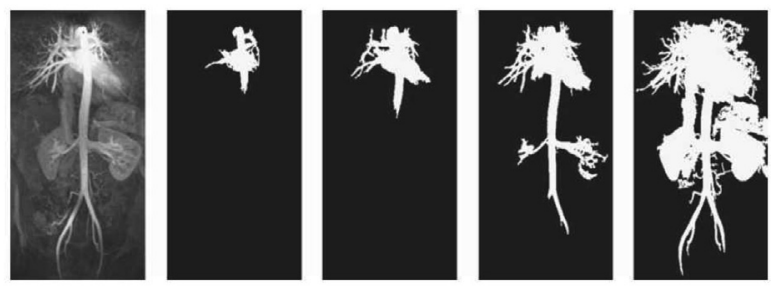

Fig. 4. Region Growing 기법은 한 점에서 시작되어 유사한 영역까지 확장해나간다. ${ }^{20}$

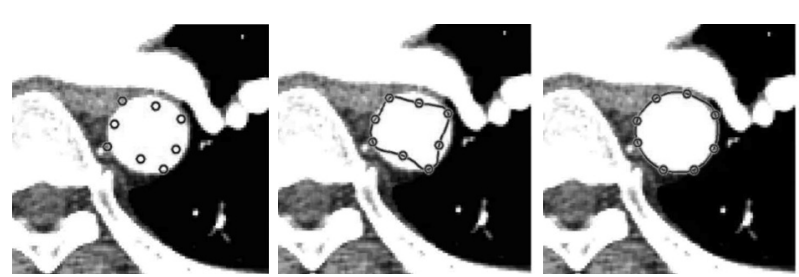

Fig. 5. Active Contour 기법은 사용자로부터 초기 경계 주위 점을 입 력 받아 각 점이 에너지가 최소화 되는 방향으로 이동해나간다.
함수가 그리는 경계가 분할 개체의 경계라고 생각하며, 0보다 작을 경우 분할 개체의 내부, 0 보다 클 경우 분할 개체의 외부라 고 생각한다. 초기 Implicit 함수는 보통 사용자로부터 입력 받 은 개체 근처의 경계를 0 , 경계 내부로 들어갈수록 점차적으로 작아지고 경계 밖으로 나아갈수록 점차적으로 커지도록 설정해 준다. 매 반복마다 점 단위가 아닌 함수단위로 갱신되면서, Active Contour와는 달리 연결되어 있지 않아도 여러 개의 개체 를 동시에 분할 할 수 있다. 하지만 Level-set에서의 Implicit Function의 함수 값은 모든 픽셀 수준에서 구하기 때문에, 처 리 시간이 길며 최대 픽셀 수준의 정밀도만을 지니는 단점이 있 다. Graph-cut 기반 분할은 모든 픽셀들이 그래프의 꼭지점으 로 보고, 에너지가 최소화 될 수 있도록 그래프를 자름으로써 원하는 대상을 추출해낸다 (Fig. 7). 모든 이웃하는 꼭지점들은 모서리로 연결되며, 모서리의 비용은 이웃 픽셀간의 값이 차이 가 크면 작아진다. 또한 모든 픽셀들은 꼭지점 $s, t$ 와 연결되어

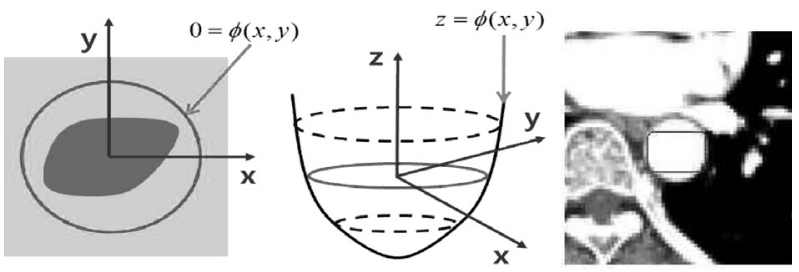

(a) 초기 함수

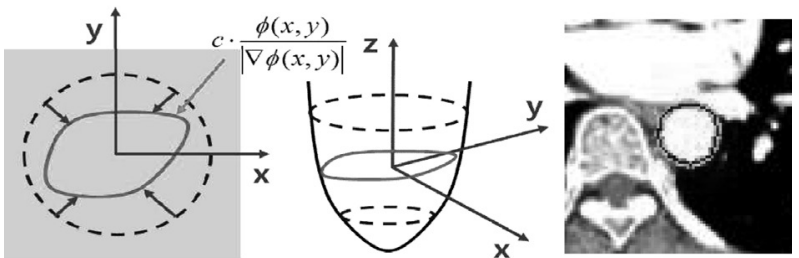
(b) Iteration을 통해 업데이트 된 함수

Fig. 6. Level Set은 implicit function과 $x-y$ 평면이 구성하는 경계가 추출 대상이며,매 iteration 마다 에너지가 최소화 되도록 implicit function이 변화한다. (a)초기 함수.

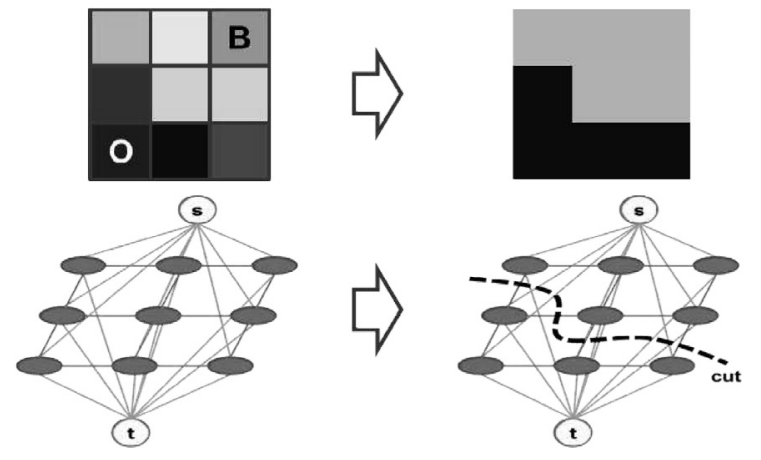

Fig. 7. Graph cut은 source node와 terminal node가 각 픽셀이 모 서리로 연결되고, 각 이웃 픽셀간에 모서리로 연결된다. 각 모서리의 값은 에너지에 따라 정의되며, Graph cut을 수행하게 되면, 각 모서 리의 에너지가 최소가 되도록 잘리게 된다. 
야 한다. 꼭지점 $t$ 와 연결된 모서리의 비용은 분할하길 원하는 개체와 통계적 특성이 유사할수록 작아지며, 꼭지점 $\mathrm{S}$ 와 연결된 모서리의 비용은 분할하고 싶지 않은 배경과 통계적 특징이 유 사할수록 작아진다. Graph-cut이 수행되면 에너지가 가장 작 은 모서리를 끊어서, 꼭지점 $\mathrm{S}$ 와 연결된 픽셀들과 꼭지점 $\mathrm{t}$ 와 연 결된 꼭지점들로 나누어지게 되는데 꼭지점 $\mathrm{s}$ 와 연결된 픽셀들 이 분할된 개체가 된다. 다시 말해서 개체와 통계적 특징이 비슷 하며, 배경과 통계적 특징이 비슷한 픽셀들이 꼭지점 $\mathrm{s}$ 와 연결되 며 끊어진 모서리는 이웃 픽셀들과 차이가 큰 경계가 된다.

위의 방법들 중 하나로 추출하길 원하는 개체의 영역을 얻으 면, 이를 3 차원으로 가시화 하기 위해서는 개체의 경계 점들을 면으로 변경하는 과정이 필요하다. 물체가 3 차원으로 보이는 이 유는 광원 (Illumination)에 따른 물체의 음영 (Shading)이 드 러나기 때문인데, 가장 널리 이용되는 Phong의 광원 모델에 따 르면 음영은 빛의 위치, 방향과 경계의 법선 벡터에 의해서 결정 된다. ${ }^{21}$ 하지만 경계 점들에는 법선 벡터가 존재하지 않기 때문 에 해당 점들을 면으로 변경 해주는 폴리곤화 (Polygonization) 과정이 필수적이다. 의료 영상처리 분야에서 가장 널리 사 용되는 폴리곤화 방법은 Marching Cube 이다. 볼륨에서 분할

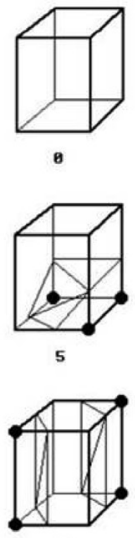

10

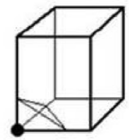

1

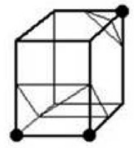

6

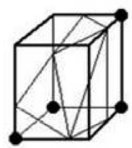

11

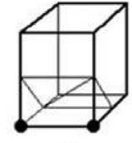

2

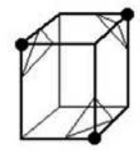

7

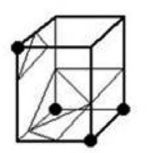

12

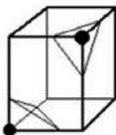

3

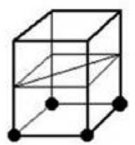

8

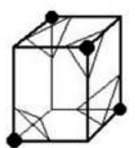

13
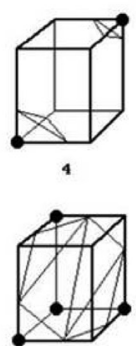

9

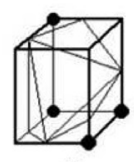

14
Fig. 8. Marching Cube는 각 큐브에서 값이 어떻게 배치되었는지에 따라서 도형을 구성한다.
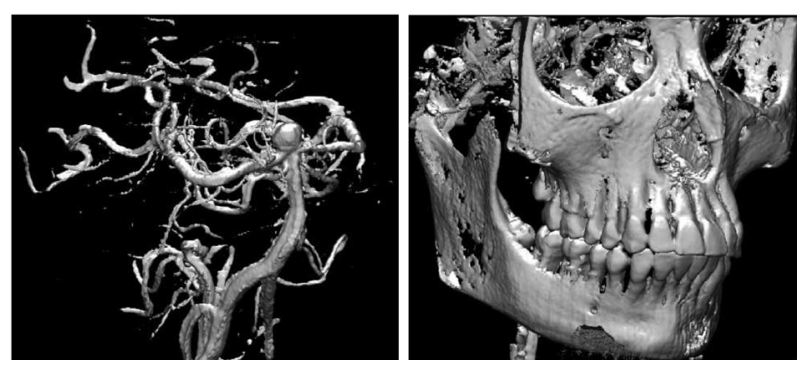

Fig. 9. Marching Cube를 이용해서 만든 폴리곤들을 3차원으로 가시 화. 21
방법을 수행하게 되면, 추출된 대상은 1 , 추출되지 않은 배경은 0으로 이루어진 마스크 볼륨이 생성되게 된다. 볼륨은 복셀로 이루어진 정육면체 큐브들이 모여서 이루어진다고 볼 수 있는 데, 큐브의 어떠한 꼭지점이 분할되었느냐에 따라 분류하게 되 면 총 256 가지 $\left(2^{8}\right)$ 의 경우의 수가 나오게 된다. 하지만 반전과 회전에 의한 중복을 제외하면 경우의 수가 14 가지로 줄게 된다. 반전에 의한 중복이란 예를 들면, 큐브의 8 꼭지점 모두 분할된 경우와 8 꼭지점 모두 분할되지 않은 경우 각각 개체의 내부, 외 부지만 이 두 가지 경우 모두 경계 면이 아니기 때문에 같은 경 우로 볼 수 있다. 이렇게 14 가지 경우의 수에 대해서만 폴리곤을 미리 정의해놓을 경우 경계를 폴리곤으로 변환시킬 수 있다 (Fig. 8). 이러한 과정을 거친 폴리곤 경계면의 법선 벡터로는 볼륨의 차분 값을 사용하게 된다.

이렇게 분할, 폴리곤화를 거친 후 3 차원으로 가시화하는 등 위면 렌더링 방법은 직접 볼륨 렌더링과 같이 계층 관계에 있는 두 개체를 동시에 가시화할 수는 없지만, 개체의 경계 정보를 가 지고 있기 때문에 폴리곤의 재배치를 통해서 분할된 개체가 더 욱 부드럽고 자연스럽게 보일 수 있도록 할 수 있어 교육용이나 전시용으로 많이 사용된다. 또한 물리모델과 결합하여 수술시의 절개, 봉합 등을 표현할 수 있으며 가상 수술 등 다양한 의학적 활용이 가능하다.

\section{결 론}

논 논문에서는 의료 영상을 3 차원으로 가시화 하는 두 가지 기술 - 직접 볼륨 렌더링, 등위면 렌더링 방법 - 에 대해서 살 펴보았다. 의료 영상을 3 차원으로 가시화 하는 방법은 영상 처 리, 광원 모델, 컴퓨터 그래픽스 등이 유기적으로 통합된 일련의 과정이다. 가시화 하길 원하는 개체를 용도에 맞게 효과적으로 가시화 하는 것은 교육, 수술 리허설, 수술 전 환자 특이적 특징 파악 등 의학 분야에서 매우 중요하다. 이러한 3 차원 가시화를 위한 유기적인 흐름에 대한 이해는 의료 영상의 활용 분야를 넓 히고 목적에 맞게 더욱 효과적으로 가시화 하기 위한 기술개발 에 도움이 될 것으로 기대한다.

\section{Acknowledgements}

본 논문은 대한민국 정부에 의한 ETRI R\&D 프로그램 [14ZC1400, 환자특이적 수술 플래닝 기반실감 리허설 기술 개발] 지원 사업의 연구 결과로 수행되었음. 


\section{참 고 문 헌}

1. Preim, B., \& Oeltze, S. (2008). 3D visualization of vasculature: an overview. In Visualization in medicine and life sciences (pp. 3959). Springer Berlin Heidelberg

2. McAuliffe, M. J., Lalonde, F. M., McGarry, D., Gandler, W., Csaky, K., \& Trus, B. L. (2001). Medical image processing, analysis and visualization in clinical research. In Computer-Based Medical Systems, 2001. CBMS 2001. Proceedings. 14th IEEE Symposium on (pp. 381-386). IEEE

3. Pham, D. L., Xu, C., \& Prince, J. L. (2000). Current methods in medical image segmentation 1 . Annual review of biomedical engineering, 2(1), 315-337

4. Prasantha, H. S., Shashidhara, H. L., Murthy, K. N. B., \& Madhavi, L. G. (2010). Medical image segmentation. International Journal on Computer Science and Engineering, 2(4), 1209-1218

5. Kim, Y. M., Baek, S. E., Lim, J. S., \& Hyung, W. J. (2013). Clinical application of image-enhanced minimally invasive robotic surgery for gastric cancer: a prospective observational study. Journal of Gastrointestinal Surgery, 17(2), 304-312

6. Kumano, S., Tsuda, T., Tanaka, H., Hirata, M., Kim, T., Murakami, T., et al. (2007). Preoperative evaluation of perigastric vascular anatomy by 3 -dimensional computed tomographic angiography using 16-channel multidetector-row computed tomography for laparoscopic gastrectomy in patients with early gastric cancer. Journal of computer assisted tomography, 31(1), 93-97

7. Matsuki, M., Kani, H., Tatsugami, F., Yoshikawa, S., Narabayashi, I., Lee, S. W., et al. (2004). Preoperative assessment of vascular anatomy around the stomach by $3 \mathrm{D}$ imaging using MDCT before laparoscopy-assisted gastrectomy. American Journal of Roentgenology, 183(1), 145-151

8. Miyaki, A., Imamura, K., Kobayashi, R., Takami, M., Matsumoto, J., \& Takada, Y. (2012). Preoperative assessment of perigastric vascular anatomy by multidetector computed tomography angiogram for laparoscopy-assisted gastrectomy. Langenbeck's Archives of Surgery, 397(6), 945-950

9. Natsume, T., Shuto, K., Yanagawa, N., Akai, T., Kawahira, H., Hayashi, H., et al. (2011). The classification of anatomic variations in the perigastric vessels by dual-phase $\mathrm{CT}$ to reduce intraoperative bleeding during laparoscopic gastrectomy. Surgical endoscopy, 25(5), 1420-1424

10. Usui, S., Hiranuma, S., Ichikawa, T., Maeda, M., Kudo, S. E., \& Iwai, T. (2005). Preoperative imaging of surrounding arteries by threedimensional CT: is it useful for laparoscopic gastrectomy? Surgical Laparoscopy Endoscopy \& Percutaneous Techniques, 15(2), 6165

11. Röttger, S., Kraus, M., \& Ertl, T. (2000, October). Hardware-accelerated volume and isosurface rendering based on cell-projection. In Proceedings of the conference on Visualization'00 (pp. 109-116). IEEE Computer Society Press

12. Parker, S., Shirley, P., Livnat, Y., Hansen, C., \& Sloan, P. P. (1998, October). Interactive ray tracing for isosurface rendering. In Proceedings of the conference on Visualization'98 (pp. 233-238). IEEE Computer Society Press

13. Drebin, R. A., Carpenter, L., \& Hanrahan, P. (1988, June). Volume rendering. In ACM Siggraph Computer Graphics (Vol. 22, No. 4, pp. 65-74). ACM

14. Lorensen, W. E., \& Cline, H. E. (1987, August). Marching cubes: A high resolution 3D surface construction algorithm. In ACM Siggraph Computer Graphics (Vol. 21, No. 4, pp. 163-169). ACM

15. Bloomenthal, J. (1988). Polygonization of implicit surfaces. Computer Aided Geometric Design, 5(4), 341-355

16. Ohtake, Y., Belyaev, A., Alexa, M., Turk, G., \& Seidel, H. P. (2005, July). Multi-level partition of unity implicits. In ACM SIGGRAPH 2005 Courses (p. 173). ACM

17. Surazhsky, V., \& Gotsman, C. (2003, June). Explicit surface remeshing. In Proceedings of the 2003 Eurographics/ACM SIGGRAPH symposium on Geometry processing (pp. 20-30). Eurographics Association

18. Sifri, O., Sheffer, A., \& Gotsman, C. (2003). Geodesic-based surface remeshing. In In Proc. 12th Intnl. Meshing Roundtable

19. Hadwiger, M., Kniss, J. M., Rezk-Salama, C., \& Weiskopf, D. (2006). Real-time volume graphics (pp. I-XVII). Natick: Ak Peters

20. Dougherty, G. (2009). Digital image processing for medical applications. Cambridge University Press

21. Smistad, E., Elster, A. C., \& Lindseth, F. (2011). Fast surface extraction and visualization of medical images using opencl and gpus. In The Joint Workshop on High Performance and Distributed Computing for Medical Imaging (Vol. 2011) 